\section{Diabetes: A fish story}

\section{By Kai-Jye Lou, Staff Writer}

Researchers at the University of California, San Diego have uncovered a mechanism that could contribute to the therapeutic effects of GlaxoSmithKline plc's Lovaza, a blockbuster fish oil extract of $\omega$ - 3 fatty acids. It is marketed as an adjunct for dieting to decrease serum triglyceride levels in patients with hypertriglyceridemia, a condition with multiple causes including diabetes. The team found that $\omega$-3 fatty acids exert their anti-inflammatory and insulin-sensitizing effects through $G$ proteincoupled receptor $120 .^{1}$

The findings could rekindle interest in $\mathrm{G}$ protein-coupled receptor 120 (GPR120) as a target for diabetes, which has been passed over in favor of its cousins, free fatty acid receptor 1 (FFAR1; GPR40) and GPR119.

GPR120 initially garnered interest as an obesity and diabetes target following publication of a 2005 paper in Nature Medicine showing that the receptor triggered the release of glucagon-like peptide-1 (GLP-1) from intestinal cells when stimulated by unsaturated long-chain free fatty acids. $^{2}$

"Our findings should give
encouragement to the
general approach of finding
an anti-inflammatory that
would improve glucose
metabolism and insulin
sensitivity." $\quad$-Jerrold Olefsky,
University of California,
San Diego

However, according to Jerrold Olefsky, associate dean for scientific affairs and a professor of medicine at UCSD, companies dismissed GPR120 as a potential therapeutic target for diabetes after follow-up work suggested its direct effect on GLP-1 release would be minor or undetectable.

Because little else was known about GPR120's physiological function, the receptor was dropped in favor of its cousins, GPR40 and GPR119, both of which have been shown to stimulate insulin secretion. ${ }^{3}$

Now, a research group led by Olefsky has uncovered additional GPR120 signaling pathways and linked the receptor to the known antiinflammatory and insulin-sensitizing effects of $\omega-3$ fatty acids.

The group surveyed a family of fatty acid-sensing GPCRs and found that GPR120 was highly expressed in mature adipocytes and proinflammatory macrophages. In the macrophages, GPR120 stimulation with $\omega$-3 fatty acids or a small molecule agonist decreased chemically induced inflammatory responses compared with no stimulation.

In adipocytes, ligand activation of GPR120 increased glucose uptake compared with small interfering RNA-mediated knockout of GPR120. Moreover, in wild-type mice fed a high-fat diet, $\omega$-3 fatty acids inhibited inflammation and reversed the loss of insulin sensitivity compared with those in GPR120 knockout mice.

The team also undertook a series of siRNA studies to map out the underlying pathway. They showed that GPR120 signaling in proinflamma- tory macrophages stimulates the association of arrestin $\beta 2$ (ARRB2) with MAP3K7 binding protein 1 (TAB1), which prevents TAB1 from activating MAP kinase kinase kinase 7 (MAP3K7; TAK1. See Figure 1, "Blocking the inflammatory pathway to insulin resistance through GPR120").

Results were published in Cell.

"Without TAB1, TAK1 cannot be activated and the inflammatory signaling pathways in these macrophages are shut down," said Olefsky, who is corresponding author on the paper and also a founder and head of the scientific advisory board at diabetes company Metabolex Inc. "Our findings should give encouragement to the general approach of finding an anti-inflammatory that would improve glucose metabolism and insulin sensitivity."

"The finding that activation of GPR120 leads ultimately to inhibition of a kinase, TAK1, should allow for a more complete understanding of the signaling pathways controlled by this receptor at the molecular level," added Noel Morgan, director of the Institute of Biomedical \& Clinical Science and a professor of endocrine pharmacology at the Peninsula College of Medicine \& Dentistry.

Metabolex's MBX-2982 is a small molecule GPR119 agonist that triggers the release of GLP-1 from intestinal $\mathrm{L}$ cells and insulin from pancreatic islet cells. It is in Phase II testing to treat type 2 diabetes. In June, the company granted sanofiaventis Group exclusive, worldwide rights to develop and commercialized MBX-2982 and several undisclosed preclinical compounds.

"Our results would also suggest that GPR120 itself could be a good target given that the antiinflammatory effects mediated through GPR120 are very robust and the receptor is highly tissue specific in its expression," said Olefsky.

"The work provides a very detailed characterization of the GPR120 signaling pathway and provides a mechanism of action that explains why $\omega$-3 fatty acids have an anti-inflammatory effect and improve insulin sensitivity," said Vincent Poitout, a professor of medicine at the University of Montreal and the Canada Research Chair in Diabetes and Pancreatic $\beta$-cell Function. "When you identify a pathway, you can definitely start to think about new points for therapeutic intervention."

\section{Worth another look}

Although the previous studies of GPR120's therapeutic potential in diabetes focused on its ability to improve GLP-1 secretion, the data in the Cell paper could prompt companies to re-examine the target as an antiinflammatory and insulin sensitizer.

"We don't yet know this for sure, but insulin sensitization through GPR120 could have fewer side effects than thiazolidinediones," noted Alan Saltiel, director of the Life Sciences Institute and a professor in the Division of Molecular Genetics at the University of Michigan. "Fish oils are widely used as a supplement and generally considered to be safe."

GSK's Lovaza is the only FDA-approved fish oil extract, and many other formulations of the oil are sold as nutraceuticals. 
Figure 1. Blocking the inflammatory pathway to insulin resistance through GPR120. Increased production of proinflammatory cytokines decreases insulin sensitivity in adipose tissues. Researchers at the University of California, San Diego found that $\omega$-3 fatty acid signaling through G protein-coupled receptor 120 (GPR120) can restore insulin sensitivity by blocking the production of proinflammatory cytokines in macrophages.

Proinflammatory M1 macrophages express toll-like receptor 4 (TLR4) and tumor necrosis factor (TNF) receptors on their surface, which can be activated by saturated fatty acids and TNF- $\alpha$, respectively [a(1)].

Signaling through these receptors via their respective ligands stimulates the production of inflammatory cytokines through pathways controlled by MAP kinase kinase kinase 7 (MAP3K7; TAK1) and its activator, MAP3K7 binding protein 1 (TAB1) [a(2)]. The secretion of these inflammatory cytokines from the macrophages leads to further macrophage activation [a(3)] and lower insulin sensitivity [a(4)].

The UCSD researchers showed that activation of GPR120 with $\omega-3$ fatty acids promotes the association of the receptor with arrestin $\beta 2$ (ARRB2), which forms a complex that is internalized into the cell $[\mathbf{b}(\mathbf{1})]$. Once internalized, the GPR120-ARRB2 complex associates with TAB1. This association prevents TAB1 from activating TAK1 $[\mathbf{b}(2)]$, which then shuts down the associated inflammatory pathways.

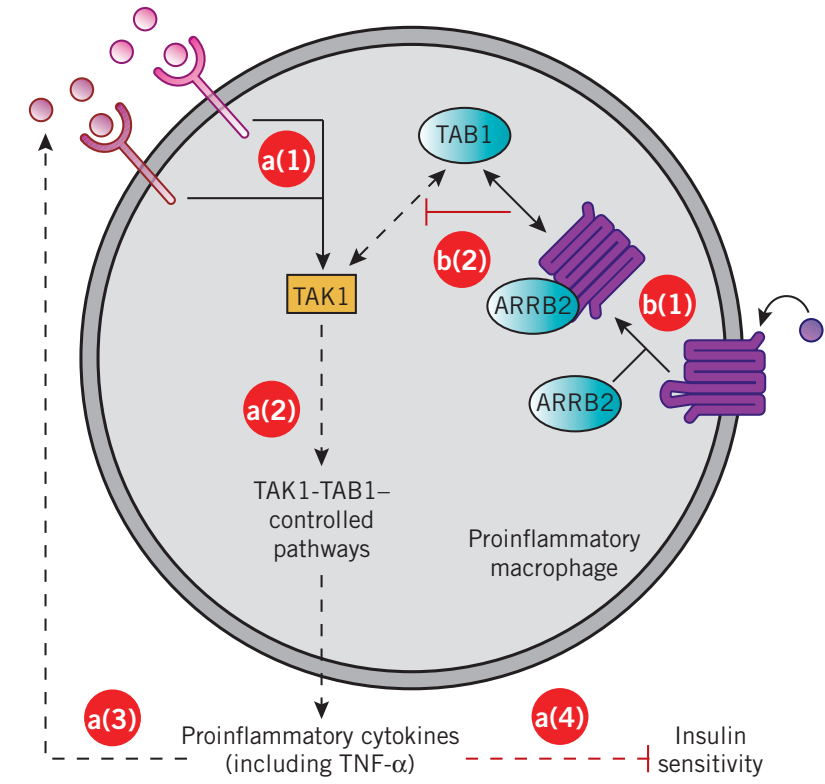

$\omega-3$ fatty acids

TNF- $\alpha$

Saturated fatty acids
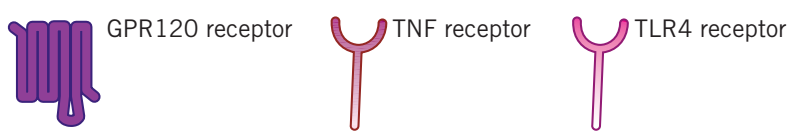

The two marketed insulin sensitizers for type 2 diabetes, Avandia rosiglitazone and Actos pioglitazone, are thiazolidinedione-based agonists of peroxisome proliferation-activated receptor- $\gamma$ (PPARG; PPAR $\gamma$ ). Both drugs have side effects including significant weight gain, edema and an increased risk for bone fracture, and Avandia has a black box warning of increased risk for congestive heart failure and myocardial ischemia.

GlaxoSmithKline markets Avandia and Takeda Pharmaceutical Co. Ltd. markets Actos.

Cristina de Min, CMO at NovImmune S.A., said it will be necessary to evaluate the $\omega-3$ fatty acids in a well-controlled clinical trial for type 2 diabetes. She noted that in patients with type 2 diabetes, $\omega-3$ fatty acid supplementation has yet to show a clear therapeutic effect against the disease.

"It will be necessary to determine whether the beneficial effects of $\omega$-3 fatty acids are strong enough to be considered a disease-modifying therapeutic effect in type 2 diabetes," de Min told SciBX.

NovImmune's NI-0401, a human mAb targeting CD3, is in Phase II testing for type 1 diabetes and Crohn's disease (CD).

Saltiel thinks the lack of therapeutic effect could be because $\omega-3$ fatty acids have not been tested at high enough doses in humans.
"It will be necessary to determine whether the beneficial effects of $\omega-3$ fatty acids are strong enough to be considered a disease-modifying therapeutic effect in type 2 diabetes."

- Cristina de Min, NovImmune S.A.

\section{An attractive target}

GPR120 has multiple properties that make it an attractive therapeutic target, such as good tissue specificity and druggability.

"Almost half of all currently used drug targets are G protein-coupled receptors," said Gozoh Tsujimoto, professor in the Department of Genomic Drug Discovery at Kyoto University. "These drug targets are located on the cell surface and usually have good tissue specificity and are usually easy to access with a low molecular weight compound."

Tsujimoto was corresponding author on the 2005 Nature Medicine paper that led to the deorphanization of GPR120.

"Our work showed that the tissue distribution of GPR120 is very restricted and highly expressed in proinflammatory macrophages and mature adipocytes but not anti-inflammatory macrophages and preadipocytes," said Olefsky. "The fact that this receptor is found in a specific subpopulation of macrophages suggests that targeting this GPR120 won't wipe out the rest of the immune system."

Saltiel nevertheless wanted to see tissue-specific GPR120 knockout studies in mice to clarify the physiological role and effects of GPR120 signaling in other tissues. And Morgan said that it may also be important to determine whether GPR120 is susceptible to downregulation or desensitization from prolonged activation. 


\section{ANALYSIS}

Poitout thinks GPR120 is likely to be druggable but also noted that it isn't clear how one should go about targeting the receptor.

"Because the GPR120 ligands identified in this study are natural compounds found in our diets, one wonders whether or not you will even need to develop a drug against this target," he said. "In fact, you may not need an agonist for GPR120, but instead a positive allosteric modulator. For example, you could give a patient the GPR120 agonist in the form of dietary $\omega$-3 fatty acids and then give the compound to enhance the effect of these fatty acids on GPR120."

Morgan also thinks that ligand specificity could be a concern.

"Although there is evidence that small molecules may hit this receptor, further work will need to be undertaken to ensure that newly developed molecules do not also bind to other fatty acid receptors, thereby adding potential complications to their actions," he told SciBX. "The evidence so far seems to imply that fatty acid receptors display a rather wide structural specificity and that they will tolerate considerable structural variability. Hence, it may prove to be more difficult to develop highly specific ligands than one would hope."

Olefsky said his group is now studying the detailed mechanisms of GPR120 signaling. "We want to map out phosphorylation sites and get the crystal structure and also study how $\omega$-3 fatty acids inhibit the migration of proinflammatory macrophages into tissue," he said.

Olefsky added that his team is planning a clinical study to evaluate pharmaceutical-grade fish oils in patients with diabetes.

"We want to know if there is a practical dose of fish oils that we can give to patients to get the right concentration of $\omega-3$ fatty acids into the blood to have a therapeutic effect in diabetes," he told SciBX.

Tsujimoto suggested looking to PPAR $\gamma$ agonists for insights on the design of new GPR120 agonists. "PPAR $\gamma$ agonists are closely related to free fatty acids in terms of structure, so they could be a good starting point for the design of new agonists for free fatty acid receptors like GPR120," he said.

UCSD has filed a patent application covering GPR120 activation for diabetes and metabolic diseases. The work is available for licensing.

Lou, K.-J. SciBX 3(37); doi:10.1038/scibx.2010.1110

Published online Sept. 23, 2010

REFERENCES

1. Oh, D.Y. et al. Cell; published online Sept. 2, 2010;

doi:10.1016/j.cell.2010.07.041

Contact: Jerrold M. Olefsky, University of California, San Diego, La Jolla, Calif.

e-mail: jolefsky@ucsd.edu

2. Hirasawa, A. et al. Nat. Med. 11, 90-94 (2005)

3. Kebede, M.A. et al. Diabetes Obes. Metab. 11, 10-20 (2009)

COMPANIES AND INSTITUTIONS MENTIONED

GlaxoSmithKline plc (LSE:GSK; NYSE:GSK), London, U.K.

Kyoto University, Kyoto, Japan

Metabolex Inc., Hayward, Calif.

Novlmmune S.A., Geneva, Switzerland

Peninsula College of Medicine \& Dentistry, Plymouth, U.K. sanofi-aventis Group (Euronext:SAN; NYSE:SNY), Paris, France

Takeda Pharmaceutical Co. Ltd. (Tokyo:4502), Osaka, Japan

University of California, San Diego, La Jolla, Calif.

University of Michigan, Ann Arbor, Mich.

University of Montreal, Montreal, Quebec, Canada 